\title{
A utilização da toxina botulínica no tratamento da paralisia facial periférica
}

\author{
The use of botulinum toxin in the treatment of peripheral facial palsy \\ El uso de la toxina botulínica en el tratamiento de la parálisis facial periférica
}

Recebido: 06/07/2021 | Revisado: 09/07/2021 | Aceito: 09/07/2021 | Publicado: 21/07/2021

\author{
Mariana Martins Andalécio \\ ORCID: https://orcid.org/0000-0002-7722-8199 \\ Centro Universitário de Patos de Minas, Brasil \\ E-mail: marianamartins@unipam.edu.br \\ Rodrigo Soares de Andrade \\ ORCID: https://orcid.org/0000-0001-6114-0929 \\ Centro Universitário de Patos de Minas, Brasil \\ E-mail: rodrigosa@unipam.edu.br \\ Luisa Carolina Santos Lima \\ ORCID: https://orcid.org/0000-0002-2284-2968 \\ Centro Universitário de Patos de Minas, Brasil \\ E-mail: luisacsl@unipam.edu.br \\ Thiago de Amorim Carvalho \\ ORCID: https://orcid.org/0000-0003-1153-0931 \\ Centro Universitário de Patos de Minas, Brasil \\ E-mail: thiagocarvalho@ unipam.edu.br \\ Ivania Aparecida Pimenta Santos Silva \\ ORCID: https://orcid.org/0000-0001-7012-8497 \\ Centro Universitário de Patos de Minas, Brasil \\ E-mail: ivaniapimenta@unipam.edu.br
}

\begin{abstract}
Resumo
A Paralisia Facial Periférica (PFP) é um estado de paralisação ou enfraquecimento dos músculos da face que causam limitações motoras e estéticas ao paciente com esta condição. Um meio de tratamento para a PFP que vem ganhando destaque é a utilização de toxina botulínica no lado contralateral ao acometido pela condição. Esta toxina é coletada através dos esporos da bactéria Clostridium botulinum, e atua inibindo a liberação de acetilcolina na junção neuromuscular, diminuindo hiperfunção muscular e devolvendo simetria a face do paciente. Portanto, este estudo tem como objetivo alcançar o conhecimento do melhor protocolo de aplicação da toxina na paralisia, além de qual sorotipo usar e a capacidade de melhoria na qualidade de vida do paciente. O estudo consta com uma revisão integrativa de caráter básico, qualitativa, descritiva-explicativa com base nas principais plataformas de pesquisa buscando os termos paralisia facial e toxina botulínica. Foram incluídos artigos no intervalo de tempo de 2011 a 2021 na língua inglesa e a busca resultou em 17 artigos. Em todos os artigos revisados o sorotipo A da toxina botulínica foi o único utilizado para o tratamento, a quantidade de unidades biológicas (UI) e o protocolo foi individualizado para cada paciente isentando de um padrão. A qualidade de vida dos pacientes foi devolvida após se obter melhora na simetria facial e com isso aumento na autoestima além de principalmente, devolver a capacidade de realizar ações musculares coordenadas. Portanto, pode-se concluir que o tratamento da PFP com toxina botulínica é altamente benéfico aos pacientes.
\end{abstract}

Palavras-chave: Paralisia facial; Toxina botulínica; Simetria; Assimetria.

\begin{abstract}
Peripheral Facial Palsy (PFP) is a state of paralysis or weakening of the facial muscles that causes motor and aesthetic limitations to the patient with this condition. One means of treatment for PFP that is gaining prominence is the use of botulinum toxin on the side contralateral to that affected by the condition. This toxin is collected from the spores of the bacterium Clostridium botulinum, and its action inhibits the release of acetylcholine at the neuromuscular junction, reducing muscle hyperfunction and restoring symmetry to the patient's face. Therefore, this study aims to achieve the knowledge of the best protocol for the application of the toxin in paralysis, as well as which serotype to use and the ability to improve the patient's quality of life. The study consists of a basic, qualitative, descriptiveexploratory integrative review based on the main research platforms searching for the terms facial paralysis and botulinum toxin. Articles were included from 2011 to 2021 in the English language and the search resulted in 17 articles. In all reviewed articles the botulinum toxin serotype A was the only one used for the treatment, the amount of biological units (BIU) and the protocol was individualized for each patient exempting from a standard. The quality of life of the patients was restored after an improvement in facial symmetry was obtained and, with this, an increase in self-esteem, besides, mainly, restoring the ability to perform coordinated muscular actions. Therefore, it can be concluded that the treatment of PFP with botulinum toxin is highly beneficial to patients.
\end{abstract}


Keywords: Facial palsy; Botulinum toxin; Symmetry; Asymmetry.

\section{Resumen}

La parálisis facial periférica (PFP) es un estado de parálisis o debilitamiento de los músculos faciales que provoca limitaciones motoras y estéticas al paciente que la padece. Un medio de tratamiento para la PFP que ha ido ganando importancia es el uso de toxina botulínica en el lado contralateral al afectado por la afección. Esta toxina se obtiene de las esporas de la bacteria Clostridium botulinum, y actúa inhibiendo la liberación de acetilcolina en la unión neuromuscular, reduciendo la hiperfunción muscular y devolviendo la simetría al rostro del paciente. Por ello, este estudio pretende lograr el conocimiento del mejor protocolo de aplicación de la toxina en la parálisis, además de qué serotipo utilizar y la capacidad de mejorar la calidad de vida del paciente. El estudio consiste en una revisión integradora de carácter básico, cualitativo, descriptivo-explicativo basada en las principales plataformas de investigación buscando los términos parálisis facial y toxina botulínica. Se incluyeron artículos en el intervalo de tiempo de 2011 a 2021 en lengua inglesa y la búsqueda dio como resultado 17 artículos. En todos los artículos revisados la toxina botulínica serotipo A fue la única utilizada para el tratamiento, la cantidad de unidades biológicas (UBI), y el protocolo fue individualizado para cada paciente eximiendo un estándar. La calidad de vida de los pacientes fue devuelta tras obtener una mejora en la simetría facial y con ello un aumento de la autoestima, además de devolver principalmente la capacidad de realizar acciones musculares coordinadas. Por lo tanto, se puede concluir que el tratamiento de la PFP con toxina botulínica es muy beneficioso para los pacientes.

Palabras clave: Parálisis facial; Toxina botulínica; Simetria; Assimetria.

\section{Introdução}

A Paralisia Facial Periférica (PFP) é um estado em que os músculos da face enfraquecem ou se paralisam, sendo resultado de algum problema do sétimo par de nervos cranianos, o nervo facial (Batista, 2011). A etiologia da paralisia facial deriva de causas diversas, como infecções, iatrogenias, síndromes, toxinas, traumatismos, tumores, acidentes vasculares cerebrais (AVCs), lesões cirúrgicas, podendo, também, ter origem congênita (Fonseca, et al. 2014). Atolini Júnior et al. (2009) e Rodrigues et al. (2014), trazem a paralisia de Bell como a etiologia mais comum, estando presente em 73,6\% dos casos do primeiro estudo e 66,6\% no segundo estudo. Esta paralisia está comumente ligada a uma reativação do Vírus Herpes Tipo I.

A toxina botulínica, extraída pela fermentação de esporos da Clostridium botulinum, é uma das toxinas bacterianas mais potentes do mundo (Dressler, et al. 2005). Ela causa uma paralisia nos músculos por meio da inibição da acetilcolina na junção neuromuscular. Possui oito sorotipos, divididos em A, B, C1, C2, D, E, F e G. Entre todos estes, o tipo A é amplamente utilizado nos procedimentos estéticos, pois possui ótima eficácia em usos corretivos e preventivos sem necessidade cirúrgica. O tipo B está sendo explorado em diversos estudos, para pacientes não respondíveis ao primeiro sorotipo (Colhado, et al. 2009; Barbosa \& Brito, 2020).

O uso da toxina botulínica na paralisia facial se dá pelo potencial em reduzir as assimetrias tanto em repouso quanto durante os movimentos voluntários e involuntários da mímica, além de melhorar a estética do paciente contribuindo para sua autoestima. Ela é utilizada do lado oposto ao acometido pela paralisia para tentar causar uma estagnação dos músculos responsáveis pelos movimentos faciais. (Maio \& Soares, 2007). Esta opção de tratamento é menos invasiva do que a opção cirúrgica também utilizada, pois possui menos riscos, não necessita de internação e não deixa cicatrizes no paciente. Além de que, se ficar insatisfatório, o tratamento é irreversível, sendo mais difícil corrigi-lo (Dobie \& Fisch, 1986).

A qualidade de vida do paciente acometido pela paralisia facial também é beneficiada pelo tratamento com a toxina botulínica, pois é possível devolver ao paciente os movimentos necessários para sorrir, falar e comer adequadamente (Heyndenrych, 2020). Segundo o estudo de Maio e Soares (2007), a satisfação com o tratamento utilizando a toxina botulínica foi muito alta, $100 \%$ dos 18 pacientes envolvidos ficaram totalmente satisfeitos com o resultado após todo o protocolo de aplicação.

Com base no exposto, o presente estudo se propõe a realizar uma revisão integrativa sobre a eficácia do uso da toxina botulínica no tratamento da paralisia facial e os benefícios causados ao paciente. Tendo como importância, avaliar a aplicabilidade da toxina botulínica em pacientes com paralisia facial periférica, analisando o melhor sorotipo da toxina para tal 
finalidade, a quantidade necessária (unidades biológicas - UI) para haver uma melhora perceptível no paciente e comprovar, através dos estudos, a melhora da qualidade de vida do paciente, visto que a toxina botulínica está cada vez mais presente neste tipo de tratamento e que é uma opção menos invasiva.

\section{Metodologia}

Este é um estudo do tipo bibliográfico, descritivo-explicativo, com análise integrativa e qualitativa de caráter básico (Estrela, 2018).

\subsection{Estratégias de Busca}

Foi realizada uma busca eletrônica nas plataformas Pubmed (MEDLINE), Scielo, e Google Schoolar, por artigos dos anos de 2011 a 2021, com base nos descritores: "Facial Palsy" e "Botulinum Toxin".

\subsection{Critérios de Inclusão e Exclusão}

Os critérios de inclusão para encontrar os artigos que foram utilizados na pesquisa são: (i) artigos que abordem a utilização da toxina botulínica exclusivamente para o tratamento de paralisia facial; (ii) artigos publicados de 2011 a 2021; (iii) artigos na língua inglesa que abranjeram o tema.

Os critérios de exclusão foram definidos por: (i) artigos que abordaram o uso da toxina botulínica em outro segmento; (ii) artigos fora do intervalo de tempo definido para a pesquisa; (iii) artigos de outro idioma a não ser na língua inglesa; (iv) artigos que sejam revisões de literatura.

Foram incluídos relatos de caso e estudos de coorte retrospectivos e prospectivos, que abordaram análises do uso da toxina botulínica em pacientes com paralisia facial de acordo com melhora estética e de qualidade de vida e, também, aqueles que trouxeram estudos sobre os melhores sorotipos e quantidade necessária para realizar o tratamento.

Com base nos critérios citados e após remoção dos artigos que não encaixavam no intuito da pesquisa (Fluxograma Figura 1), foram encontrados 17 artigos que serão apresentados a seguir nos resultados.

\section{Resultados}

A busca nas plataformas pelos termos: "facial palsy" e "botulinum toxin" identificaram um total de 339 artigos na língua inglesa. Logo após, foram aplicados os critérios de exclusão. Depois de filtrar o intervalo de tempo dos últimos dez anos, o resultado da busca foi para 183 artigos. Pela leitura dos títulos e resumos dos artigos, foram excluídos os artigos em duplicata ou sem acesso e também aqueles que não se enquadraram no tema do estudo, restando 17 artigos que se enquadraram nos critérios de inclusão propostos (Figura 1).

Realizou-se então, a revisão por meio da leitura em integra destes 17 artigos e seus principais dados foram destacados na tabela abaixo (Tabela 1). Em sua maioria foram estudos de coorte prospectivos e retrospectivos e casos clínicos que abrangiam o uso da toxina botulínica para o tratamento exclusivo de paralisia facial periférica trazendo seus benefícios e protocolos utilizados por cada autor. 
Figura 1: Fluxograma do processo de busca na literatura.

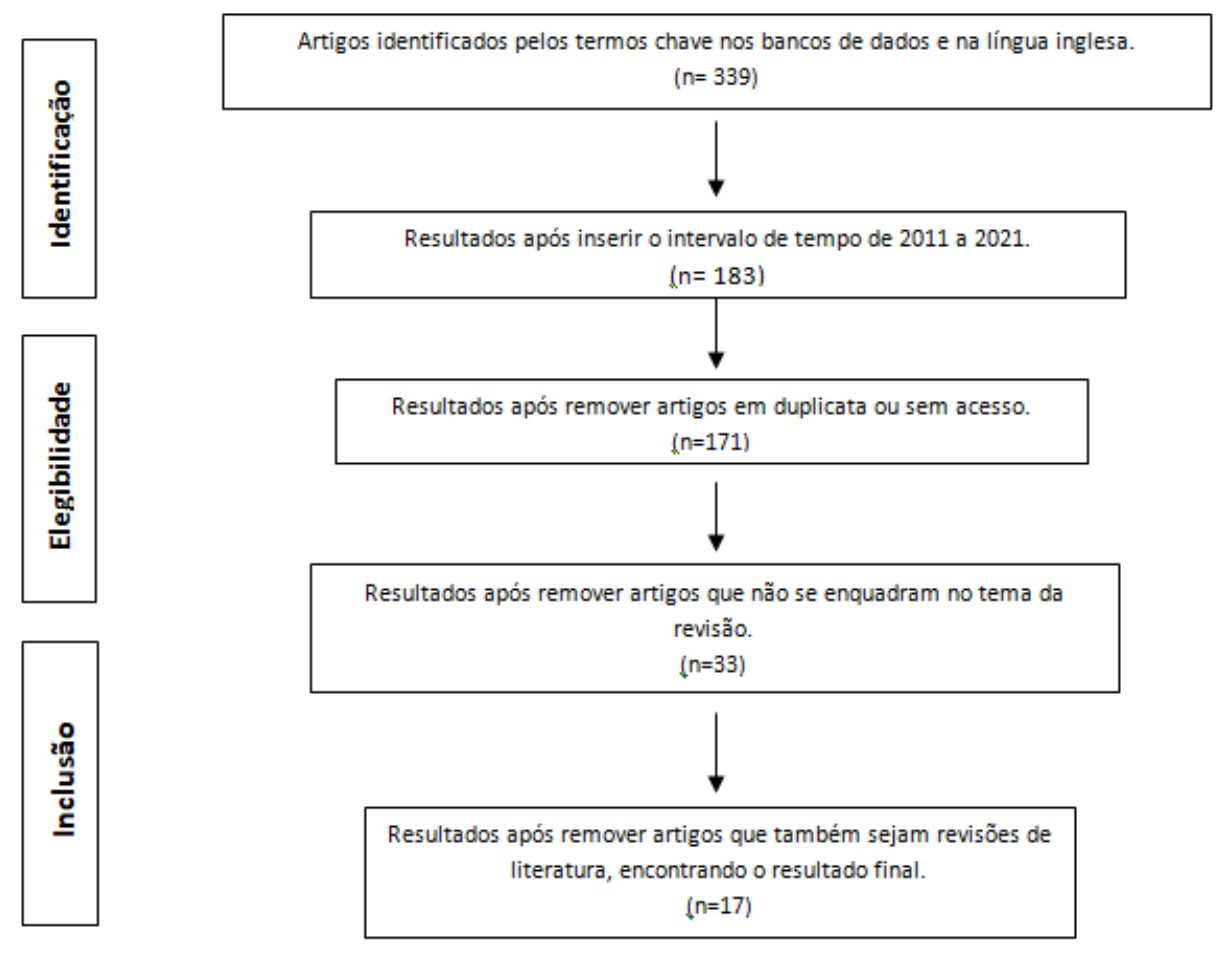

Fonte: Autores.

Tabela 1: Artigos utilizados na revisão integrativa sobre a utilização de toxina botulínica no tratamento de paralisia facial periférica mostrando o sorotipo usado, dose e resultados encontrados pelos autores.

\begin{tabular}{|l|l|l|l|l|l|l|l|}
\hline Autores & País & $\begin{array}{l}\text { Tipo de } \\
\text { Estudo }\end{array}$ & Pac. & $\begin{array}{l}\text { Quantidade de } \\
\text { Toxina }\end{array}$ & Sorotipo & IT & Resultado \\
\hline $\begin{array}{l}\text { Carvalho } \\
\text { et al., 2019 }\end{array}$ & Brazil & $\begin{array}{l}\text { Estudo de } \\
\text { Coorte } \\
\text { Prospectivo }\end{array}$ & 40 & $\begin{array}{l}12 \text { a } 60 \text { UI } \\
+-35 \text { UI/paciente }\end{array}$ & BONT-A & $\begin{array}{l}15 \text { a } \\
180 \\
\text { dias }\end{array}$ & $\begin{array}{l}\text { Impacto positivo. } \\
\text { Resultado mais } \\
\text { rápido em mulheres. }\end{array}$ \\
\hline $\begin{array}{l}\text { Neville et al., } \\
2017\end{array}$ & EUA & $\begin{array}{l}\text { Questionário } \\
\text { SAQ) }\end{array}$ & 51 & $\begin{array}{l}0,5 \text { a 5 UI por } \\
\text { ponto de injeção }\end{array}$ & BONT-A & $\begin{array}{l}16 \text { a } \\
140 \\
\text { dias }\end{array}$ & $\begin{array}{l}\text { Efeitos positivos } \\
\text { significativos. } \\
\text { Mais de um ciclo de } \\
\text { injeção por paciente. }\end{array}$ \\
\hline $\begin{array}{l}\text { Bennis Y., } \\
\text { Duquennoy- } \\
\begin{array}{l}\text { Martinot V., } \\
\text { Guerreschi P., } \\
\text { 2016. }\end{array}\end{array}$ & França & $\begin{array}{l}\text { Epidemiologic } \\
\text { Overview for } \\
11 \text { years. }\end{array}$ & 50 & $\begin{array}{l}9,8 \text { UI para lado } \\
\text { saudável } \\
10,4 \text { para lado } \\
\text { paralisado } \\
\text { por paciente }\end{array}$ & BONT-A & $\begin{array}{l}\text { Efeitos positivos } \\
\text { significativos. Em } \\
\text { ambos os lados } \\
\text { utilizados, o objetivo } \\
\text { foi alcançado. }\end{array}$ \\
\hline
\end{tabular}




\begin{tabular}{|c|c|c|c|c|c|c|c|}
\hline $\begin{array}{l}\text { Shinn et al., } \\
2019 .\end{array}$ & EUA & $\begin{array}{l}\text { Estudo de } \\
\text { Coorte } \\
\text { Prospectivo } \\
\text { (Questionário - } \\
\text { SAQ) }\end{array}$ & 99 & $\begin{array}{l}2 \text { a } 3 \text { UI para cada } \\
\text { músculo tratado } \\
\text { (exceto platisma - } \\
9 \text { a } 10 \text { UI). }\end{array}$ & BONT-A & & $\begin{array}{l}\text { Efeitos positivos. } \\
\text { Melhor resposta em } \\
\text { mulheres, jovens e } \\
\text { casos avançados. } \\
\text { Maior dose = maior } \\
\text { resposta. }\end{array}$ \\
\hline $\begin{array}{l}\text { Alipour et al., } \\
2021\end{array}$ & Alemanha & $\begin{array}{l}\text { Análise } \\
\text { Retrospectiva } \\
\text { de } 1998 \text { a } \\
2018 .\end{array}$ & 73 & $\begin{array}{l}\text { OnaBont A e } \\
\text { IncoBont A: 24UI } \\
\text { AboBont A: 69UI }\end{array}$ & BONT-A & & $\begin{array}{l}\text { Efeitos positivos } \\
\text { secundários a } \\
\text { paralisia. } \\
\text { Foi tratado o lado } \\
\text { paralisado e o } \\
\text { oposto. }\end{array}$ \\
\hline $\begin{array}{l}\text { Mandrine et al., } \\
2016\end{array}$ & Itália & $\begin{array}{l}\text { Estudo de série } \\
\text { de casos. }\end{array}$ & 27 & $\begin{array}{l}15 \text { a } 21 \text { UI por } \\
\text { sessão }\end{array}$ & BONT-A & & $\begin{array}{l}\text { Efeitos positivos na } \\
\text { motilidade facial } \\
\text { voluntária associado } \\
\text { a reabilitação de } \\
\text { espelho. }\end{array}$ \\
\hline $\begin{array}{l}\text { Sahan A., } \\
\text { Tamer F., } 2017 .\end{array}$ & Turquia & Relato de Caso & 1 & $6,5 \mathrm{UI}$ & BONT-A & & $\begin{array}{l}\text { Correção de } \\
\text { Assimetria pela } \\
\text { Paralisia Facial } \\
\text { causada após } \\
\text { cirurgia de orelha } \\
\text { média. }\end{array}$ \\
\hline $\begin{array}{l}\text { Akulov et al., } \\
2017\end{array}$ & Rússia & $\begin{array}{l}\text { Estudo de } \\
\text { Coorte. }\end{array}$ & 76 & $\begin{array}{l}\text { Fase Aguda da } \\
\text { paralisia: } \\
\text { 40-50 UI por } \\
\text { paciente. } \\
\text { Fase Crônica: } \\
\text { 1- 1,5 UI por ponto } \\
\text { de injeção. }\end{array}$ & BONT-A & & $\begin{array}{l}\text { Aliado a exercícios } \\
\text { de fisioterapia houve } \\
\text { melhorias } \\
\text { significativas após } 3 \\
\text { meses de tratamento. }\end{array}$ \\
\hline $\begin{array}{l}\text { Rutzen et al., } \\
2016 .\end{array}$ & Porto Rico & $\begin{array}{l}\text { Relato de Caso } \\
\text { Clínico }\end{array}$ & 1 & $\begin{array}{l}45 \text { UI em todo o } \\
\text { tratamento }\end{array}$ & BONT-A & $\begin{array}{l}6 \\
\text { sema } \\
\text { nas }\end{array}$ & $\begin{array}{l}\text { Melhora na simetria } \\
\text { facial e diminuição } \\
\text { de mordidas labiais. }\end{array}$ \\
\hline $\begin{array}{l}\text { Pecora C. S., } \\
\text { Shitara D., } \\
2021 .\end{array}$ & Brasil & $\begin{array}{l}\text { Relato de } \\
\text { Experiência }\end{array}$ & - & $\begin{array}{l}35 \text { a } 51 \text { UI por } \\
\text { paciente }\end{array}$ & BONT-A & $\begin{array}{l}2 \\
\text { sema } \\
\text { nas }\end{array}$ & $\begin{array}{l}\text { Eficaz na devolução } \\
\text { da simetria facial no } \\
\text { lado não afetado, } \\
\text { redução de espasmos } \\
\text { no lado afetado. }\end{array}$ \\
\hline $\begin{array}{l}\text { Diaz-Aristizabal } \\
\text { et al., } 2021\end{array}$ & Espanha & $\begin{array}{l}\text { Estudo de } \\
\text { Coorte } \\
\text { Prospectivo }\end{array}$ & 20 & $\begin{array}{l}7,5 \text { a } 100 \text { UI } \\
\text { Média de } 50 \text { UI. }\end{array}$ & BONT-A & $\begin{array}{l}4 \\
\text { sema } \\
\text { nas }\end{array}$ & $\begin{array}{l}\text { Aumento na } \\
\text { funcionalidade } \\
\text { facial, melhora na } \\
\text { qualidade de vida e } \\
\text { diminuição da } \\
\text { assimetria facial. }\end{array}$ \\
\hline $\begin{array}{l}\text { Choi K. H., Rho } \\
\text { S. H., Lee J. M., } \\
\text { Jeon J. H., Park } \\
\text { S. Y., Kim J., } \\
\text { 2013. }\end{array}$ & Coreia & $\begin{array}{l}\text { Estudo Clínico } \\
\text { Prospectivo }\end{array}$ & 42 & $\begin{array}{l}\text { Lado Paralisado: } \\
\text {-por ponto } 1,5 \mathrm{a} \\
2,5 \mathrm{UI} \\
\text {-por paciente } 10 \mathrm{a} \\
26 \mathrm{UI} \\
\text { Lado Normal: } \\
\text {-por ponto } 2,5 \text { a } 5 \\
\text { UI } \\
\text {-por paciente } 35 \text { a } \\
72 \text { UI. }\end{array}$ & BONT-A & & $\begin{array}{l}\text { Melhora na } \\
\text { qualidade de vida, } \\
\text { aparência e para a } \\
\text { injestão de alimento. } \\
\text { Aumento na força } \\
\text { facial do lado } \\
\text { paralisado. }\end{array}$ \\
\hline $\begin{array}{l}\text { Remígio A. F. } \\
\text { N., Salles A. G., } \\
\text { Faria J. C. M, }\end{array}$ & Brasil & Estudo Clínico & 55 & $\begin{array}{l}\text { Por paciente: } \\
\text {-Onabotulinum } 15 \\
\text { a } 70 \text { UI. }\end{array}$ & BONT-A & $\begin{array}{l}1 \text { e } 6 \\
\text { mese } \\
\text { s }\end{array}$ & $\begin{array}{l}\text { Diminuição da } \\
\text { Assimetria. }\end{array}$ \\
\hline
\end{tabular}




\begin{tabular}{|c|c|c|c|c|c|c|c|}
\hline $\begin{array}{l}\text { Ferreira M. C., } \\
2014 .\end{array}$ & & & & $\begin{array}{l}\text {-Abobotulinum } 16 \\
\text { a } 64 \text { UI. }\end{array}$ & & & \\
\hline Kim, 2013. & Coreia & Estudo Clínico & 18 & $\begin{array}{l}\text { Por ponto: } 2,5 \mathrm{a} \\
\text { 3UI. } \\
\text { Por paciente: } 32 \mathrm{a} \\
68 \text { UI. }\end{array}$ & BONT-A & $\begin{array}{l}1 \text { e } 6 \\
\text { mese } \\
\text { s }\end{array}$ & $\begin{array}{l}\text { Redução da } \\
\text { Hiperfunção do lado } \\
\text { não paralisado, } \\
\text { devolvendo simetria } \\
\text { durante os } \\
\text { movimentos faciais. }\end{array}$ \\
\hline $\begin{array}{l}\text { Lee J. M., Choi } \\
\text { K. H., Lim B. } \\
\text { W., Kim M. W., } \\
\text { Kim J., } 2014 .\end{array}$ & Coreia & $\begin{array}{l}\text { Estudo de } \\
\text { Coorte } \\
\text { Prospectivo }\end{array}$ & 17 & $\begin{array}{l}\text { Por ponto: } 1,5 \text { a } 3 \\
\text { UI. }\end{array}$ & BONT-A & $\begin{array}{l}2 \\
\text { anos }\end{array}$ & $\begin{array}{l}\text { Combinação com } \\
\text { Biofeedback no } \\
\text { espelho: alívio da } \\
\text { sincinésia e redução } \\
\text { da assimetria facial. }\end{array}$ \\
\hline $\begin{array}{l}\text { Risoud M, } \\
\text { Aljudaibi N., } \\
\text { Duquennoy- } \\
\text { Martinot V., } \\
\text { Guerreschi P., } \\
2015 .\end{array}$ & França & Estudo Clínico & 30 & $\begin{array}{l}\text { Lado não } \\
\text { paralisado: } \\
\text {-por ponto } 2,8 \text { a } \\
6,25 \text { UI } \\
\text {-por paciente +/- } \\
39 \text { UI } \\
\text { Lado paralisado: } \\
\text {-por ponto } 2 \text { a } 9 \text { UI } \\
\text {-por paciente +/- } \\
37 \text { UI. }\end{array}$ & BONT-A & $\begin{array}{l}2 \text { a } 3 \\
\text { anos }\end{array}$ & $\begin{array}{l}\text { Melhora na } \\
\text { hiperatividade } \\
\text { muscular e na } \\
\text { simetria facial. }\end{array}$ \\
\hline $\begin{array}{l}\text { Sadiq S. A., } \\
\text { Khwaja S., } \\
\text { Saeed S. R., } \\
2012 .\end{array}$ & Itália & Estudo Clínico & 14 & $\begin{array}{l}\text { Média de } 30 \mathrm{UI} \\
\text { por paciente. } \\
\text { Variando de } 10 \text { a } \\
80 \text { UI. }\end{array}$ & BONT-A & $\begin{array}{l}6 \\
\text { dias }\end{array}$ & $\begin{array}{l}\text { Melhoria na } \\
\text { simetria, no } \\
\text { entendimento da fala } \\
\text { pelos familiares. } \\
\text { Aumento da } \\
\text { confiança. }\end{array}$ \\
\hline
\end{tabular}

IT: intervalo de tempo entre as sessões, nos artigos em que utilizou-se de mais de uma aplicação. BONT-A: Toxina Botulínica Tipo A.

Fonte: Autores.

\section{Discussão}

A terapêutica da paralisia facial periférica (PFP) utilizando-se de toxina botulínica tem como função reduzir ou eliminar as ações involuntárias dos músculos da face no lado paralisado e diminuir assimetria e hiperfunção dos músculos no lado não paralisado ou contralateral (Cabin, Massry e Azizzadeh, 2015). Isto é possível pela capacidade que a toxina botulínica tem de inibir a liberação de acetilcolina nas terminações nervosas motoras, diminuindo a contração muscular (Ribeiro et al., 2014).

Após analisar a literatura sobre este tema, é possível concluir que não há um padrão no protocolo de tratamento. Os autores relatam que as doses são específicas para cada paciente, já que dependem de sexo, tempo em que a pessoa já se encontra com paralisia, etiologia, idade e resposta do organismo. Neville et al. (2017) trouxeram que os sintomas relatados pelos pacientes é outro dado que deve ser observado antes de se concluir o plano de tratamento. Portanto, o protocolo deve ser individualizado e definido após examinar cada paciente.

A quantidade de unidades biológicas da toxina botulínica varia tanto de paciente para paciente quanto de músculo para músculo, como descrito na tabela 1. Um exemplo disso pode ser encontrado no estudo de Kim (2013). A dose utilizada em cada paciente neste estudo variou de 32 a 68 U. Esta quantidade utilizada foi definida após a examinação do grupo de músculos envolvidos na paralisia facial em cada paciente, pois quanto mais músculos envolvidos, maior a dose necessária para se atingir um resultado favorável.

A quantidade máxima de unidades biológicas internacionais (UI) utilizadas por paciente, esteva presente no estudo de Sadiq, Khwaja e Saeed (2012) sendo de 80 UI, porém neste mesmo estudo houve pacientes em que se utilizou somente 10 UI. 
Pode-se concluir mais uma vez com esta informação que o protocolo deve ser criado com base no paciente, portanto não há uma dose padrão que pode ser utilizada em todos os tratamentos.

Se compararmos a diferença da dose por ponto (músculo) no estudo de Shinn, et al. (2019) há uma diferença de 2 a 3 unidades biológicas de um para outro, com exceção do músculo platisma que pode chegar a até 10 UI. Logo, podemos reparar que alguns músculos acometidos pela paralisia facial necessitam de uma maior quantidade de toxina para responderem como o esperado. Além do platisma citado anteriormente, o orbicular da boca e o elevador do lábio também necessitam de uma maior quantidade de material.

Outros estudos trouxeram quantidades bem diferentes de toxina por pontos de injeção. Choi, et al. (2013) realizou a aplicação de 1,5 a 2,5 UI no lado paralisado e 2,5 a 5 UI por ponto no lado contralateral. Lee, et al. (2014) realizou um estudo de coorte prospectivo em que os pacientes receberam em média três sessões de aplicação da toxina em doses de 1,5 a 3 UI por músculo. Já o estudo de Akulov, et al. (2017) foi o único que apresentou a utilização da toxina em diferentes fases da paralisia facial. Na fase aguda (24 a 48 horas após a paralisia), as doses variaram de 1 a 4 UI por músculo do lado contralateral e a aplicação foi repetida após quatro meses. Na fase crônica (oito meses após paralisia) a dose variou-se de 1 a 1,5 UI por músculo do lado afetado e foi utilizada também no lado contralateral com o dobro da dose em cada ponto. Houve melhoras significativas em ambas às fases de aplicação, portanto o tratamento, segundo este estudo, pode sim ser benéfico em ambos os estágios.

Em todos os artigos encontrados após a pesquisa, os autores relatam a melhora na simetria e capacidade motora facial dos pacientes acometidos pela paralisia facial periférica após o tratamento com a toxina botulínica. Rutzen, et al. (2016) trouxe ainda que os pacientes relataram diminuição de mordidas na mucosa do lado paralisado. Estes estudos ressaltam a melhora na qualidade de vida do paciente que passa a ser capaz de realizar movimentos faciais mais coordenados e em equilíbrio com o lado contralateral, sendo assim, a toxina botulínica está sendo considerada por diversos autores como um novo meio de tratamento para esta condição de forma menos traumática que a cirúrgica e com um pós-imediato.

Outro achado comum apresentado em todos os artigos foi o uso do sorotipo A no tratamento, sendo o padrão chave na escolha da toxina, pois traz os melhores resultados estéticos. Os outros sorotipos da toxina não foram comentados por nenhum dos autores, salientando que o tipo A é realmente o mais utilizado para esta finalidade em unanimidade.

Remígio, et al. (2014) e Alipour, et al. (2021) trouxeram em seus estudos as diferentes formulações de preparo do sorotipo A, sendo eles Onabont, Incobont e Abobont, o que alterou no uso, apenas a quantidade de unidades biológicas utilizadas por cada formulação, porém a Abobont foi causadora de fraqueza em alguns pacientes após a injeção. Além destes autores, Sadiq, et al. (2012) também observou efeito colateral de hipersalivação em três de seus 14 pacientes. Porém a melhoria no entendimento da fala, na simetria e o aumento de confiança relatado pelos pacientes comprova que este tratamento tem mais benefícios que malefícios.

Shinn, et al. (2019) trouxe a Paralisia de Bell como a etiologia mais frequente para a paralisia facial periférica seguido de sequela cirúrgica na ressecção de Schwamoma vestibular. Ainda com relação a dados estatísticos, a maioria dos pacientes dos estudos são mulheres numa média de 45 a 66 anos, podendo indicar uma prevalência de acometimento no gênero feminino e na fase adulta. Alipour, et al. (2021) relatou em seu estudo, que a etiologia da paralisia facial interfere tanto na dosagem quanto na frequência e resultado do tratamento.

Já a resposta ao tratamento é mais rápida em mulheres, jovens e pacientes com a gravidade da paralisia mais avançada (Shinn, et al., 2019). Em todos os estudos comparados, houve mais de uma sessão de aplicação da toxina por paciente, e a cada sessão, a dose era elevada até atingir sua fase estacionária ou de melhor resposta. Com exceção ao estudo de Kim (2013) em que foi realizado apenas uma aplicação por paciente. O intervalo de tempo entre as sessões variou-se de seis semanas (Rutzen, et al., 2016), três meses (Alipour, et al., 2021) e quatro meses (Akulov, et al., 2017). 
Choi, et al. (2013), Risoud, et al. (2015), Bennis, Duquennoy-Martinot e Guerresch (2016), Akulov, et al. (2017), Shinn et al. (2019), em seus estudos seguiram o protocolo de tratamento associando a injeção da toxina botulínica tanto no lado paralisado para reduzir espasmos e melhorar a força, quanto do lado não paralisado para diminuir hiperatividade muscular e reduzir assimetria. Segundo estes autores, a combinação de tratamento em ambos os lados possui maior eficácia sendo considerado o melhor padrão de tratamento. Em relação à quantidade utilizada em cada lado, foi relatado uma dose duas vezes maior no lado não paralisado. Choi, et al. (2013) observou no resultado de seu estudo um aumento da força facial do lado paralisado após a injeção no lado contralateral, por reduzir a força do lado não afetado e chamou de Fenômeno de Redistribuição de Forças.

Mandrine (2016) e Akulov, et al. (2017) utilizaram de um treinamento neuromuscular facial com espelho ou biofeedback de eletromiografia como complementação ao tratamento com toxina botulínica. Os pacientes foram orientados por profissionais para realizar três expressões (rosnar, sorrir e franzir os lábios) diariamente na frente de um espelho. Estes exercícios ajudaram na criação de uma melhor memória motora nos pacientes e em movimentos mais coordenados e simétricos. Em ambos os estudos os resultados do treinamento ajudaram a se obter uma resposta satisfatória para o tratamento dos pacientes.

O tratamento da PFP utilizando de toxina botulínica foi visto por todos os autores como benéfico ao paciente e com resultados satisfatórios, portanto deve-se dar importância ao estudo do tema e a inserção deste tipo de apoio aos pacientes que se encontram com esta condição instalada. Tanto a autoestima quanto a condição motora dos indivíduos são gravemente afetados pela consequência da paralisia, portanto é de suma importância conseguir devolver a estes pacientes a possibilidade de realizar suas ações de maneira ordenada além de sua qualidade de vida. A individualidade deste tipo de tratamento, ressaltada pelos autores é um ponto importante do estudo, pois devemos dar a atenção necessária à particularidade de cada caso.

\section{Conclusão}

Nesta revisão integrativa pôde-se concluir que o sorotipo da toxina botulínica utilizado no tratamento da paralisia facial periférica é o sorotipo $\mathrm{A}$ em unanimidade entre os autores. Com relação à quantidade necessária de unidades biológicas (UI) da toxina para se realizar o melhor protocolo de tratamento, é findado que não existe um valor ideal, por depender dos fatores envoltos no estado do paciente individualmente, como sexo, idade, etiologia da paralisia facial periférica, tempo em que se encontra com a condição e quantidade de músculos envolvidos. A qualidade de vida dos pacientes que realizam este tipo de tratamento é notoriamente melhorada em todos os artigos revisados, visto que os pacientes recuperam seus movimentos faciais coordenados, capacidade de comunicação, expressão e melhora na assimetria, com isso a autoestima é recuperada.

Estudos futuros com abordagens em tentar identificar um protocolo clínico que possa ser seguido utilizando da toxina botulínica de maneira mais padronizada entre os pacientes podem ser feitos para que haja um norte onde os profissionais sigam e consigam alcançar um tratamento mais efetivo e duradouro para a paralisia facial periférica.

\section{Referências}

Akulov, M. A., Orlova, O. R., Orlova, A. S., Usachev, D. J., Shimansky, V. N., Tanjashin, S. V., Khatkova, S. E. \& Yunosha- Shanyavskaya, A. V. (2017). IncobotulinumtoxinA treatment of facial nerve palsy after neurosurgery. Journal of the Neurological Sciences, (381), $130-134$.

Alipour, S., Pick, C., Jansen, S. Rink, S. Klubmann, J. P., \& Grosheva, M. (2021). Long-term therapy with botulinum toxin in facial synkinesis: Retrospective data analysis of data from 1998 to 2018. Clinical Otolaryngology published by John Wiley \& Sons Ltd., 00:1-9.

Atolini Junior, N.,Jorge Junior, J. J., Gignon, V. F., Kitice, A. T., Prado, L. S. A. \& Santos, V. G. W. (2009). Paralisia Facial Periférica: Incidência das Várias Etiologias num Ambulatório de Atendimento Terciário. Arq. Int. Otorrinolaringol., 13(2), 167-171.

Barbosa, D. B. M. \& Brito, A. S. (2020). A utilização da toxina botulínica tipo a para alcançar a estética facial. Revista Terra \&amp;amp; Cultura: Cadernos de Ensino e Pesquisa, 36(70),75-86. 
Batista, K. T. (2011). Paralisia facial: análise epidemiológica em hospital de reabilitação. Rev. Bras. Cir. Plást., 26(4), 591-595.

Bennis, Y., Duquennoy-Martinot, V. \& Guerresch, P. (2016). Epidemiologic Overview of Synkinesis in 353 Patients with Longstanding Facial Paralysis under Treatment with Botulinum Toxin for 11 years. American Society of Plastic Surgeons.

Cabin, J. A., Massry, G. G. \& Azizzadeh, B. (2015). Botulinum toxin in the management of facial paralysis, Current Opinion in Otolaryngology \&amp; Head and Neck Surgery, 23(4), 272-280.

Carvalho, V. F., Vieira, A. P. S., Paggiaro, A. O., Salles, A. G \& Gemperli, R. (2019). Evaluation of the body image of patients with facial palsy before and after the application of botulinum toxin. International Journal of Dermatology, 58(10), 1175-1183,

Choi, K. H., Rho, S. H., Lee, J. M., Jeon, J. H., Park S. Y. \& Kim, J. (2013). Botulinum toxin injection of both sides of the face to treat post-paralytic facial synkinesis. Journal of Plastic, Reconstructive \& Aesthetic Surgery, 66, 1058-1063.

Colhado, O. C. G., Boeing, M. \& Ortega, L. B. (2009). Toxina Botulínica no Tratamento da Dor. Revista Brasileira de Anestesiologia, 59(3), 366-381.

Díaz-Aristizabal, U., Valdés-Vilches, M., Fernández-Ferreras, T. R., Calero-Muñoz, E., Bienzobas-Allué, E., Aguilera-Ballester, L., \& Carnicer-Cáceres, J. (2021). Effect of botulinum toxin typeA in functionality, synkinesis and quality of life in peripheral facial palsy sequelae. Efecto de la toxina botulínica tipoA en la funcionalidad, las sincinesias y la calidad de vida en secuelas de parálisis facial periférica. Neurologia (Barcelona, Spain), S0213-4853(21)00032-3.

Dobie, R. A. \& Fisch, U. (1986). Primary and revision surgery (selective neurectomy) for facial hyperkinesia. Arch Otorynolaringol Head Neck Surg., 112(2), $154-163$.

Dressler, D., Saberi, F. A. \& Barbosa, E. R. (2005). Botulinum toxin: mechanisms of action. Arq. Neuro-Psiquiatr., 63(1), 180-185.

Estrela, C. (2018). Metodologia Científica: Ciência, Ensino, Pesquisa. Editora Artes Médicas.

Fonseca, K. M. O., Mourão, A. M., Motta, A. R. \& Vicente, L. C. C. (2015). Scales of degree of facial paralysis: analysis of agreement. Braz. j. otorhinolaryngol., 81(3), 288-293.

Heyndenrych, I. (2020). The Treatment of Facial Asymmetry with Botulinum Toxin: Current Concepts, Guidelines, and Future Trends. Indian Journal of Plastic Surgery, 53(2), 219-229.

Kim, J. (2013). Contralateral Botulinum Toxin Injection to Improve Facial Asymmetry After Acute Facial Paralysis. Otology \& Neurotology, $34,319-324$.

Lee, J. M., Choi, K. H., Lim, B. W., Kim, M. W. \& Kim, J. (2015). Half-mirror biofeedback exercise in combination with three botulinum toxin A injections for long-lasting treatment of facial sequelae after facial paralysis. Journal of Plastic, Reconstructive \& Aesthetic Surgery, 68, 71-78.

Maio, M. \& Soares, M. F. D. (2007). Toxina Botulínica em Paralisia Facial: um Tratamento Miminamente Invasivo para Redução da Hipercinesia Muscular da Região Perioral Contralateral. Arq. Int. Otorrinolaringol., 11(1), 28-35.

Mandrini, S., Comelli, M., Dall'angelo, A., Togni, R., Cecini, M., Pavese, C. \& Toffola, E.D. (2016). Long-term facial improvement after repeated BoNT-A injections and mirror biofeedback exercises for chronic facial synkinesis: a case-series study. European Journal of Physical and Rehabilitation Medicine, $52(6), 810-818$.

Neville, C., Venables, V., Aslet, M., Nduka, C. \& Kannan, R. (2017). An objective assessment of Botulinum toxin type A injection in the treatment of post facial palsy synkinesis and hyperkinesis using the Synkinesis Assessment Questionnaire (SAQ). Journal of Plastic, Reconstructive \& Aesthetic Surgery, $70(11), 1624-1628$.

Pecora, C. S. \& Shitara, D. (2021). Botulinum Toxin Type A to Improve Facial Symmetry in Facial Palsy: A Practical Guideline and Clinical Experience. Journal Toxins, 13, 159

Remígio, A. F. N., Salles, A. G., Faria, J. C. M. \& Ferreira, M. C. (2013). Comparison of the Efficacy of OnabotulinumtoxinA and AbobotulinumtoxinA at the 1:3 Conversion Ratio for the Treatment of Asymmetry after Long-Term Facial Paralysis. Plastic and Reconstructive Surgery, 135(1), $239-249$.

Ribeiro, I. N. S., Santos, A. C. O., Gonçalves, V. M. \& Cruz, E. F. (2014). O Uso da Toxina Botulínica tipo A nas Rugas Dinâmicas do Terço Superior da Face. Revista da Universidade Ibirapuera. 7, 31-37.

Risoud, M., Aljudaibi, N., Duquennoy-Martinot, V. \& Guerreschi, P. (2015). Long-term sequelae treatment of peripheral facial paralysis with botulinum toxin type A: Repartition and kinetics of doses used. Annales de chirurgie plastique esthétique.

Rodrigues, R. E. C., Ceccato, S. B., Rezende, C. E. B., Garcia, R. I. D., Costa, K. S., Campilongo, M. \& Rapoport, P. B. (2002). Paralisia facial periférica: análise de 38 casos. Fundação ABC, 27(2).

Rutzen, I. M., Atkins, D. Q. \& Motta, K. (2016). EMG Guided Botulinum Toxin Type A Injections for Functional Problems Associated to Congenital Facial Palsy: A Case Report. 261-262.

Sadiq, S. A., Khwaja, S. \& Saeed, S. R. (2012). Botulinum toxin to improve lower facial symmetry in facial nerve palsy. Macmillan Publishers Limited, 26, 1431-1436.

Sahan, A. \& Tamer, F. (2017). Restoring facial symmetry through non-surgical cosmetic procedures after permanent facial paralysis: a case report. Acta Dermatovenerol, 26, 41-42.

Shinn, J. R., Nwabueze, N. N., Du, L., Patel, P. N., Motamedi K. K., Norton, C., Ries, W. R. \& Stephane S. J. (2019). Treatment Patterns and Outcomes in Botulinum Therapy for Patients With Facial Synkinesis. JAMA Facial Plast Surg. 21(3), 244-251. 